\title{
Adjunctive intravitreal dexamethasone in the treatment of acute endophthalmitis following cataract surgery
}

\author{
Edward F Hall' \\ Garrett R Scott' \\ David C Musch'1,2 \\ David N Zacks' \\ 'Department of Ophthalmology \\ and Visual Sciences, Medical School; \\ ${ }^{2}$ Department of Epidemiology, School \\ of Public Health; University of \\ Michigan, Ann Arbor, MI, USA
}

Purpose: Controversy exists regarding the use of intravitreal dexamethasone (IVD) as an anti-inflammatory adjunct to intravitreal antibiotics in patients with acute endophthalmitis following cataract surgery. The purpose of this project was to evaluate our experience regarding the effect of adjunctive IVD use on visual outcomes in such patients.

Design: Retrospective, comparative case series.

Methods: Study population: Patients treated for acute endophthalmitis following cataract surgery from 1995-2004. Intervention: In addition to standard intravitreal antibiotic treatment, some patients also received a single adjunctive injection of IVD. Primary outcome measures: Median visual acuity at last follow-up and percentage of patients achieving a $\geq 3$-line improvement in visual acuity. Secondary outcome measures: Inflammatory index scoring, including amount of cell and flare, height of hypopyon, and presence of fibrin as a function of time after treatment.

Results: Twenty-six eyes were treated with and 38 eyes without adjunctive IVD. Median presenting visual acuity was Hand Motion in both groups. Median visual acuity at last followup measured 20/40 in the IVD group and 20/50 in the No-IVD group $(p=0.75)$. Seventy-three percent of patients in the IVD group and $82 \%$ of patients in the No-IVD group achieved $\mathrm{a} \geq 3$-line improvement in visual acuity $(\mathrm{p}=0.42$ ). No significant difference was detected between the IVD and No-IVD groups for any of the three measures of inflammation.

Conclusion: The use of IVD did not significantly improve the final median visual acuity, the chance of achieving a $\geq 3$-line improvement in visual acuity, or the amount of intraocular inflammation. Based on these findings, and the possible detrimental effect of IVD on visual outcomes previously reported in the literature, the use of IVD does not appear to be warranted as a routine adjunctive treatment in postoperative endophthalmitis.

Keywords: endophthalmitis, dexamethasone, intravitreal injection

\section{Introduction}

The Endophthalmitis Vitrectomy Study (EVS) helped provide treatment guidelines (EVSG 1995) for acute postoperative endophthalmitis, a potentially devastating complication of intraocular surgery. Although the use of intravitreal dexamethasone (IVD) was not studied in the EVS, this adjunctive treatment has gained popularity as a means to potentially decrease intraocular inflammation and thereby improve visual outcomes. In the 2004 Preference and Trends Survey of practicing retina specialists conducted by the American Society of Retina Specialists, 43\% of respondents said that they routinely use intravitreal steroids as an adjunct to intravitreal antibiotics in the treatment of endophthalmitis patients (Pollack 2006).

Despite the intuitive appeal of decreasing intraocular inflammation, the clinical benefit of this practice has yet to be validated in the literature. To date, only three studies specifically examine the effect of this adjunctive treatment on visual acuity 
outcomes, and they present conflicting results (Das et al 1999; Shah et al 2000; Gan et al 2005a). One retrospective analysis reported a detrimental effect of adjunctive IVD, with patients receiving adjunctive IVD significantly less likely to obtain a 3-line improvement in visual acuity than patients receiving intravitreal antibiotics only (Shah et al 2000). One prospective study found that visual outcomes were similar in patients treated with or without adjunctive IVD, however, certain subsets of IVD-treated patients did have a more rapid resolution of their intraocular inflammation (Das et al 1999). The third study, also prospective in nature, suggested a trend towards improved vision with IVD, though statistical significance was not achieved (Gan et al 2005a). In animal models of endophthalmitis, the benefit of IVD has varied. Some investigators have reported that IVD appears to decrease intraocular inflammation and perhaps attenuate retinal necrosis on a histopathologic basis (Maxwell et al 1991; Park et al 1995; Smith et al 1997; Yoshizumu et al 1998; Liu et al 2000; Yildrim et al 2002). Others have reported no overall benefit (Jett et al 1995; Kim et al 1996; Pollack et al 2004) and perhaps even harmful effects (Meredith et al 1996). Intravitreal dexamethasone may also affect the concentration of intravitreal vancomycin, though this too is unclear, with one study showing lower vancomycin concentrations (Smith et al 1991) and another study showing a significantly increased half-life of intravitreal vancomycin in the presence of IVD (Park et al 1999). A recent human study found that IVD did not decrease the intravitreal vancomycin concentration (Gan et al 2005b).

With the recent suggestion that endophthalmitis rates have been increasing (West et al 2005), and the equivocal and conflicting animal and human clinical studies to guide us in our use of adjunctive IVD, we sought to review our experience with the use of IVD in improving visual outcomes and decreasing inflammation in patients with acute, post-cataract endophthalmitis.

\section{Methods}

Charts of all consecutive patients diagnosed and treated by the Retina Service at the University of Michigan's Kellogg Eye Center for acute postoperative endophthalmitis following cataract surgery over the nine-year period between July 1 , 1995 and June 30, 2004 were reviewed. Institutional review board approval was obtained for this study. Patients included in the analysis presented within 30 days of cataract surgery with symptoms and signs consistent with endophthalmitis, including pain, blurred vision, anterior chamber reaction, hypopyon, and/or vitritis. Patients were excluded if there was a history of uveitis or retained lens material. All patients included in this analysis were followed for at least 30 days after initiation of endophthalmitis treatment.

Each patient was treated by a staff physician of the Kellogg Eye Center Retina Service with either one of two regimens according to EVS protocol: a vitreous tap and injection of intravitreal antibiotics, or vitrectomy and placement of intravitreal antibiotics. Antibiotic treatment consisted of vancomycin $(1 \mathrm{mg} / 0.1 \mathrm{~mL})$ with either ceftazidime $(2.25 \mathrm{mg} / 0.1 \mathrm{~mL})$ or amikacin $(400 \mu \mathrm{g} / 0.1 \mathrm{~mL})$. Some patients additionally received subconjunctival, oral, and/or topical antibiotics, at the discretion of the treating physician. The treatment variable examined in this study was the injection of IVD. The decision to inject IVD was based on treating physician preference. Two physicians used adjunctive IVD routinely, two never used adjunctive IVD, and two based the decision on "clinical severity" as determined by the physician at the time the patient was examined (with more "severe" cases receiving IVD). Assignment of the treating physician to the patient was determined according to a call schedule, and as such could be considered random. Among the patients who received IVD, each had only one injection $(400 \mu \mathrm{g} / 0.1 \mathrm{~mL})$, which occurred at the time of the treatment intervention (tap and inject or vitrectomy). Some patients in each group (those treated with IVD and those without IVD) also received a subconjunctival injection of dexamethasone, some received oral prednisone, and some received topical steroids, also at the discretion of the treating physician.

Patients treated by vitrectomy had a vitreous biopsy analyzed by sending both initial undiluted and final diluted vitreous washings for bacteriologic analysis. Patients not treated by vitrectomy underwent anterior chamber and vitreous tap. Anterior chamber tap was performed by inserting a 30-gauge needle at the limbus with aspiration of $0.1 \mathrm{~mL}$ of aqueous. Vitreous tap was performed using a 25-gauge needle inserted through the pars plana with aspiration of $0.1 \mathrm{~mL}$ of vitreous. All samples were immediately plated on culture media and sent to the Microbiology Laboratory at the University of Michigan for Gram stain, cultures, and determination of antibiotic sensitivities.

Each patient's chart was reviewed to determine age, presenting visual acuity, interval between cataract surgery and diagnosis of endophthalmitis, anterior chamber reaction (graded on $1+$ to $4+$ scale), hypopyon (measured in millimeters), presence or absence of fibrin, endophthalmitis treatment regimen (vitrectomy or tap/injection), use of IVD, infecting organism, and post-treatment visual acuity and inflammatory indices (cell/flare, hypopyon, fibrin) at all available follow-up 
visits. The primary outcome measures were visual acuity and the percentage of patients achieving a $\geq 3$-line (or category) improvement in visual acuity. Visual acuity was measured using the Snellen acuity chart. If a patient was unable to read the Snellen chart, visual acuity was tested for the ability to count fingers, recognize hand motions, or detect light in a manner similar to that used in the EVS. All acuities were converted to a $\log$ MAR scale before statistical analyses were performed. If a patient was enucleated or eviscerated, they were assigned an acuity of "no light perception" (NLP) for purposes of analysis. Visual acuities at various intervals were compared using the Mann-Whitney U-test for comparing median values and the Pearson's chi-square test for comparing proportions. Statistical significance was assumed to have been reached when the chance of a type 1 error was less than $5 \%$. Logistic regression was used to determine whether any of the factors listed above were associated with binary primary outcome measures. Repeated measures analysis was used to assess for differences in any of the inflammatory measures as a function of time after treatment between the IVD and No-IVD groups. A mixed model regression analysis was used for the cell/flare and hypopyon measures and a generalized estimating equation regression was used for the categorical index of presence or absence of fibrin. All three analyses adjusted for baseline differences between the two groups. SAS 9.1 statistical software (Cary, NC) was used for all analyses.

\section{Results}

Our series represents the largest of its kind to date, with 64 patients meeting the inclusion criteria. Of these, 26 (41\%) were treated with IVD (IVD group) and 38 (59\%) were treated without IVD (No-IVD group). The baseline characteristics were similar in both groups. The average age was 69.8 years in the IVD group and 73.2 years in the No-IVD group. The average interval between cataract surgery and diagnosis of endophthalmitis was 7.4 days in the IVD group and 7.0 days in the No-IVD group. Intraocular inflammatory scores (including grading of anterior chamber reaction, presence and height of hypopyon, and presence of fibrin in the anterior chamber) were similar in each group at presentation. A higher (but not statistically significant) percentage of patients in the IVD group were treated with vitrectomy than in the No-IVD group $(31 \%$ [8/26]) vs $16 \%$ [6/38] respectively, $p=0.15)$. In the IVD group, follow-up ranged from 30 days to 12 months with a mean of 110 days. In the No-IVD group, follow-up ranged from 30 days to 24 months with a mean of 121 days.
The distribution of presenting visual acuities for the two groups is shown in Figure 1. Median baseline visual acuity was $3.0 \log$ MAR (HM) in each group. Though the median presenting acuity was equal in the two groups, only $62 \%$ $(16 / 26)$ of patients presented with visual acuity of Hand Motion or better in the IVD group versus $84 \%$ (32/38) in the No-IVD group $(\mathrm{p}=0.04)$. The use of extravitreal (ie, oral, subconjunctival, and topical) steroids and antibiotics was similar in each group (data not shown).

A specific bacterium was identified in $73 \%(19 / 26)$ of cases in the IVD group and $66 \%(25 / 38)$ of cases in the No-IVD group (Table 1). In the IVD group, organisms included coagulase-negative Staphylococcus $(\mathrm{n}=12)$, Staphylococcus aureus $(\mathrm{n}=1)$, and Streptococcus species $(\mathrm{n}=6)$. In the No-IVD group, organisms included coagulase-negative Staphylococcus $(\mathrm{n}=14), S$, aureus $(\mathrm{n}=5)$, Streptococcus species $(\mathrm{n}=2)$, Enterococcus $(\mathrm{n}=1)$, and other $(\mathrm{n}=3)$. In some instances, the organism was identified with a nonspecific notation (eg, "Staphylococcus" or "Gram-poistive cocci") or there was no documentation of the culture result (categorized as "not identified" in Table 1).

The distribution of visual acuities at last follow-up in each group is displayed in Figure 2. The median visual acuities at last follow-up were similar at 20/40 in the IVD group and $20 / 50$ in the No-IVD group $(p=0.75)$. The percentage of patients with $\mathrm{a} \geq 3$-line improvement in visual acuity were also similar at $73.1 \%(19 / 26)$ in the IVD group and $81.6 \%$ $(31 / 38)$ in the No-IVD group $(\mathrm{p}=0.42)$. Twenty patients in the IVD group (76.9\%) had a visual acuity of $20 / 400$ or better at last follow-up compared with $31(81.6 \%)$ in the No-IVD group $(\mathrm{p}=0.65)$. Loss of vision after treatment was observed in only three patients in the IVD group and two patients in the No-IVD group.

Because there was a disparity in the distribution of presenting visual acuities between the two groups, we compared visual acuity outcomes between the IVD and No-IVD groups at each presenting visual acuity (Table 2). The data confirm the trend toward similar outcomes across presenting visual acuities, with the No-IVD group perhaps doing slightly better for most subgroups, but the numbers within each category were too small to allow for meaningful statistical analysis.

To help reduce possible confounding effects caused by differences in the virulence of various infecting strains, we performed a subgroup analysis including only patients infected with coagulase-negative Staphylococcus. The median presenting visual acuity for patients infected with coagulase-negative Staphylococcus was similar between the two groups at between CF and HM $(\mathrm{p}=0.40)$. The median 


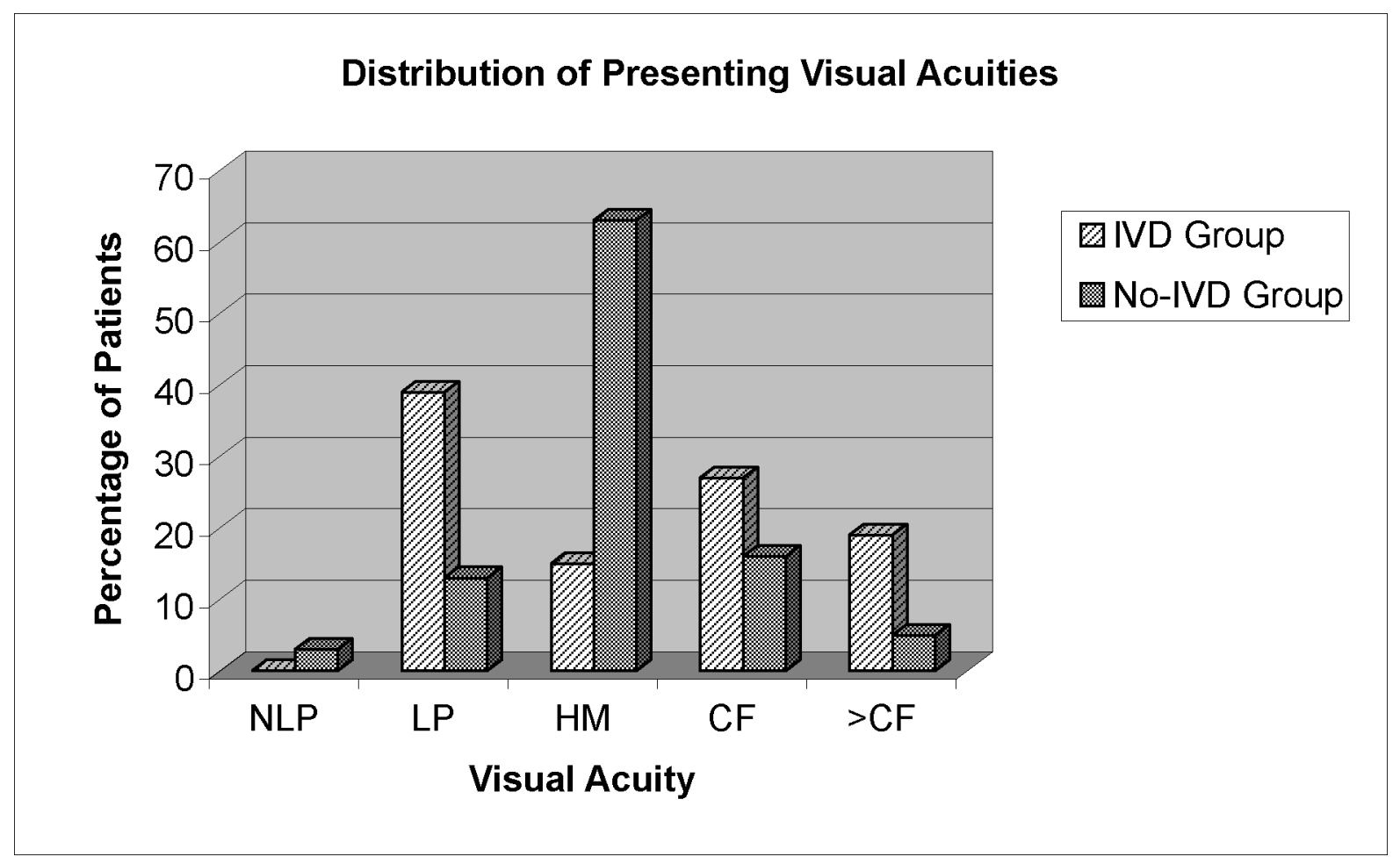

Figure I Distribution of presenting visual acuities in patients treated with or without intravitreal dexamethasone (IVD). The percentage of patients presenting with HM or better vision was significantly higher in the No-IVD group $(p=0.04)$.

final visual acuity was $20 / 40$ in the IVD group $(n=12)$ and 20/50 in the No-IVD group $(n=14)$. At last recorded follow-up for this subgroup, $\geq 3$-line improvement in visual acuity was observed in $92 \%$ (11/12) of patients in the IVD group and $100 \%(14 / 14)$ in the No-IVD group.

Possible predictive factors for an improved visual outcome ( $\geq 3$-line improvement at last follow-up) were evaluated

Table I Distribution of organisms in patients treated with or without adjunctive intravitreal dexamethasone (IVD). The Streptococcus group has been subcategorized by strains

\begin{tabular}{lll}
\hline & IVD group & No-IVD group \\
& N (\%) & n (\%) \\
\hline Not identified & $2(7)$ & $3(8)$ \\
Culture negative & $5(19)$ & $10(26)$ \\
Coag - Staphylococcus & I2 (46) & $14(37)$ \\
Staphylococcus aureus & I (4) & $5(13)$ \\
"Staphylococcus" & 0 & I (3) \\
Streptococcus & $6(23)$ & $2(5)$ \\
Intermedius & I (4) & 0 \\
Mitis & I (4) & 0 \\
Sanguis & I (4) & 0 \\
Salivarius & I (4) & 0 \\
Pneumoniae & I (4) & $2(5)$ \\
Group B Streptococcus & I (4) & 0 \\
"Gram + cocci" & 0 & $2(5)$ \\
Enterococcus & 0 & I (3) \\
\hline
\end{tabular}

individually using logistic regression. An intravitreal injection of dexamethasone ( $p=0.59)$ was not among them. Other factors that were not significantly associated with an improved visual outcome included a poor presenting visual acuity $<20 / 400(p=0.55)$, vitrectomy $(p=0.23)$, and age $(p=0.15)$. Patients whose endophthalmitis was associated with coagulase-negative Staphylococcus as the causative agent were significantly more likely to show an improved visual outcome $(\mathrm{OR}=19.1 ; 95 \% \mathrm{CI}=2.3$ to $160.1 ; \mathrm{p}=0.0065)$. Of the 26 patients whose endophthalmitis was attributed to coagulase-negative Staphylococcus, 25 (96\%) had improved visual outcomes, whereas of the 30 patients who had other bacterial causes or no organism isolated, 17 (57\%) showed improved visual outcomes (eight patients were not included because culture results either could not be determined or were nonspecific). A multiple logistic regression model confirmed that only coagulase-negative Staphylococcus was predictive of visual improvement $(\mathrm{OR}=21.7 ; 95 \% \mathrm{CI}=2.2$ to $209.1 ; \mathrm{p}$ $=0.0078$ ) upon adjustment for presenting visual acuity, use of IVD, vitrectomy, and age.

The use of adjunctive IVD did not contribute to a more rapid resolution of inflammation in our patients (Table 3). The intraocular inflammatory scores of anterior chamber reaction, height of hypopyon, and presence of fibrin remained 


\section{Distribution of Visual Acuities at Last Follow-Up}
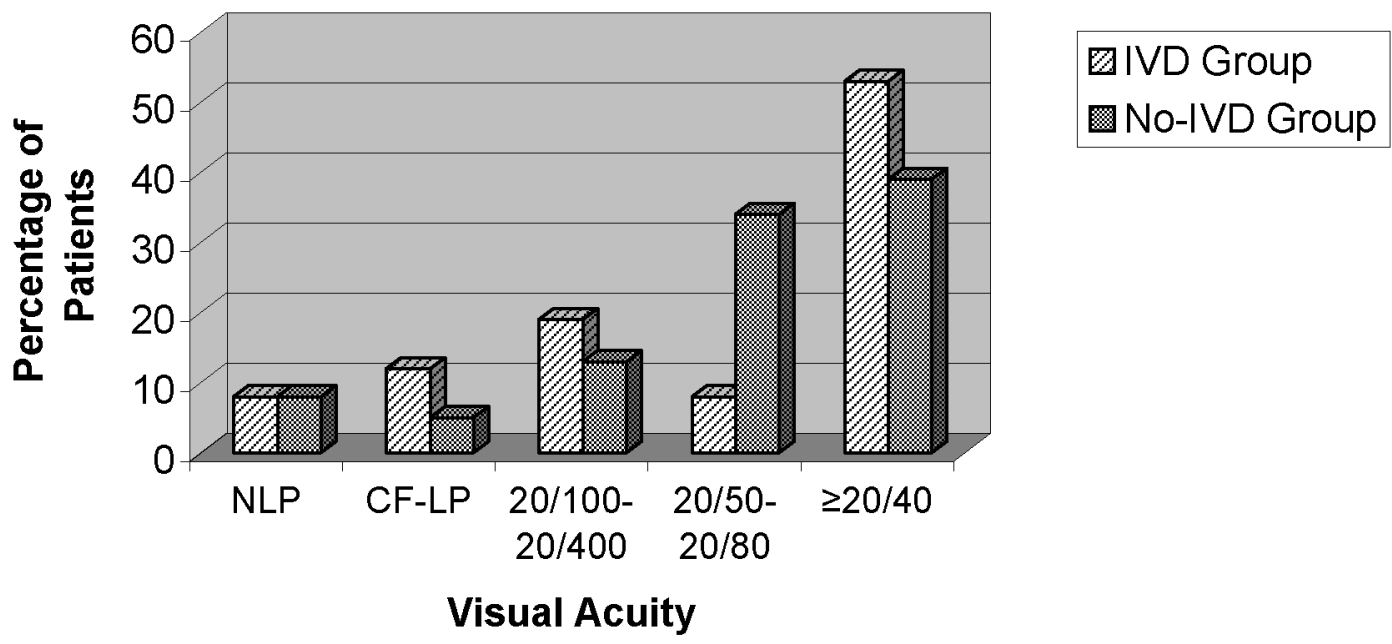

Figure 2 Distribution of visual acuities at last follow-up in patients treated with or without intravitreal dexamethasone (IVD).

similar between the two groups at various time points up to 30 days after initiation of treatment $(\mathrm{p}=0.24, \mathrm{p}=0.16$, and $\mathrm{p}=0.61$, respectively). To determine whether an overall lack of difference between groups may mask a short-term impact of IVD, we assessed the difference in each of the inflammatory indices between day 0 (day of treatment) and day 7 ( 1 week after treatment). This analysis, too, failed to detect any significant difference between the two groups.

\section{Discussion}

The data presented here represent the largest analysis to date examining the potential role of intravitreal dexamethasone as an adjunct to antibiotics in the treatment of acute, post-cataract endophthalmitis. We attempted to determine

Table 2 Median visual acuity at last follow-up when controlling for presenting visual acuities. Presenting visual acuities are displayed on the left. Final Median VA = median visual acuity at last follow-up

\begin{tabular}{lll}
\hline Presenting Va & $\begin{array}{l}\text { IVD group } \\
\text { Final median VA }\end{array}$ & $\begin{array}{l}\text { No-IVD group } \\
\text { Final median VA }\end{array}$ \\
\hline $\mathrm{LP}$ & $20 / 125-20 / 160$ & $20 / 50$ \\
$\mathrm{HM}$ & $20 / 80-20 / 100$ & $20 / 50$ \\
$\mathrm{CF}$ & $20 / 40$ & $20 / 63$ \\
$>20 / 400$ & $20 / 40$ & $20 / 25-20 / 30$ \\
\hline
\end{tabular}

the effect of adjunctive IVD on visual acuity and on the clearance of intraocular inflammation. In our population, the primary outcome measures of median visual acuity and a $\geq 3$-line improvement in visual acuity showed no significant difference between patients treated with or without IVD. Furthermore, we did not detect any significant improvement in intraocular inflammation in IVD patients relative to the No-IVD patients. Though we did not see a worse outcome in the IVD patients, as was observed in the study of Shah and colleagues (2000), our data agree with theirs in that they do not support the use of intravitreal dexamethasone as a routine adjunct to antibiotics in the treatment of post-cataract endophthalmitis.

A major limitation of our analysis, as well as that of Shah and colleagues (2000), is the retrospective nature of the study. The two additional papers on adjunctive IVD, one by Das and colleagues (1999) and another by Gan and colleagues (2005a), evaluated outcomes with adjunctive IVD through prospective randomized trials. Comparing our results with theirs is complicated by the different study designs and treatment protocols. Irrespective of these differences, however, their results also failed to demonstrate any conclusive benefit from the use of adjunctive IVD.

Our study had several limitations that might have affected our ability to detect an improved outcome in patients 
Table 3 Inflammation scores for patients treated with or without adjunctive intravitreal dexamethasone (IVD). The time points are days after treatment (Day 0 ). The $\mathrm{p}$-value represents the probability that there was a difference between the two groups over time

\begin{tabular}{|c|c|c|c|c|c|c|c|c|c|}
\hline & \multicolumn{4}{|c|}{ IVD group } & \multicolumn{4}{|c|}{ No-IVD group } & \multirow[b]{2}{*}{ p-value } \\
\hline & Day 0 & DayI & Day 7 & Day 30 & Day 0 & Day I & Day 7 & Day 30 & \\
\hline $\begin{array}{l}\text { Mean cell/flare } \\
\text { (scale of I-4) }\end{array}$ & 2.9 & 2.1 & 0.9 & 0.3 & 2.7 & 2.3 & 1.2 & 0.3 & 0.24 \\
\hline Hypopyon (mm) & 0.5 & 0.8 & 0.2 & 0 & 0.5 & 0.5 & 0.02 & 0 & 0.16 \\
\hline Fibrin present (\%) & 66 & 62 & 17 & 0 & 61 & 60 & 12 & 9 & 0.61 \\
\hline
\end{tabular}

receiving IVD. One limitation was the difference between the distribution of presenting visual acuities in the IVD and No-IVD groups. This may have been reflective of an individual physician's preference for using IVD in certain subsets of eyes (eg, worse presenting visual acuity) (Figure 1). Only $63 \%$ of our patients presented with visual acuity of HM or better in the IVD group compared with $87 \%$ in the No-IVD group, yet the median visual acuity at last follow-up and the final distributions of visual acuities are nearly identical. This could suggest that the IVD was beneficial, at least for patients with poorer baseline visions. However, neither the univariate nor multivariate analyses indicated that presenting visual acuity was predictive of response to adjunctive IVD.

Another limitation of the study is the variability in the use of topical, subconjunctival, and oral steroids and antibiotics. This type of limitation is inherent in retrospective analyses. We suspect that these adjunctive treatments have limited effect on visual outcomes when compared with intravitreal therapies.

Though a higher percentage of patients in our IVD group were treated with vitrectomy (reflecting the difference in presenting visual acuities and therefore the recommendations from the EVS), this difference was not statistically significant. Patients treated with vitrectomy might have been expected to have more rapid improvement in visual acuity because of the removal of vitreous debris, but we found no association between vitrectomy and visual acuity outcomes. We were also unable to detect a difference between the two groups in the rate of clearing of intraocular inflammation based on anterior chamber reaction scores, hypopyon height, and presence of fibrin in the anterior chamber.

One factor that may have affected our results was the distribution of infecting organisms. There was a slightly higher rate of streptococcal infection (which might be more virulent) in the group treated with IVD and a higher rate of negative cultures in the No-IVD group. The relatively small numbers preclude statistical subgroup comparison. Patients infected with coagulase-negative Staphylococcus responded similarly in each group. The finding that patients infected with coagulase-negative Staphylococcus were significantly more likely to have a $\geq 3$-line improvement in visual acuity is in agreement with results from the EVS (Johnson et al 1997), and is likely reflective of the less virulent nature of this organism.

Our study is also limited by the lack of long-term followup data regarding other secondary outcomes such as epiretinal membrane formation or need for vitrectomy to clear vitreous debris. The Retina Service at Kellogg Eye Center services a large geographic area, and patients often are returned to the care of the referring ophthalmologist when they appeared to be clinically stable and improving. Inspection of the limited data available at longer follow-up intervals revealed no substantial differences in visual acuity between the groups (data not shown).

Although Peyman and colleagues (1974) published the first report of the use of IVD in the treatment of endophthalmitis more than 30 years ago, there is still no consensus regarding the use of IVD in the treatment of endophthalmitis. Our data and those from other investigators show that IVD seems to have minimal if any significant effect on visual outcomes, and as such its routine use as an adjunct to intravitreal antibiotics does not appear warranted. A randomized clinical trial to test the benefit of adjunctive IVD would seem impractical, given the large number of patients that one would need to recruit to detect any significant effect. More research is necessary to identify the specific virulence and inflammatory mediators responsible for the damage to ocular structures in endophthalmitis as limiting such tissue destruction may not be achievable with adjunctive intravitreal steroids alone (Ermis et al 2007). Such work would help in the design of targeted adjunctive therapies specifically designed to minimize the collateral damage caused by the infection, and improve visual outcomes.

\section{Disclosure}

There are no commercial interests. Supported in part by a departmental grant from Research to Prevent Blindness, Inc. 
Presented in part at the 2005 Association for Research in Vision and Ophthalmology meeting.

\section{References}

Das T, Jalali S, Gothwal VK, et al. 1999. Intravitreal dexamethasone in exogenous bacterial endophthalmitis: results of a prospective randomised study. Br J Ophthalmol, 83:1050-5.

Ermis SS, Cetinkaya Z, Kiyici H, et al. 2007. Effects of intravitreal moxifloxacin and dexamethasone in experimental Staphylococcus aureus endophthalmitis. Curr Eye Res, 32:337-44.

[EVSG] Endophthalmitis Vitrectomy Study Group. Results of the Endophthalmitis Vitrectomy Study. 1995. A randomized trial of immediate vitrectomy and of intravitreous antibiotics for the treatment of postoperative bacterial endophthalmitis. Arch Ophthalmol, 113:1479-96.

Gan IM, Ugahary LC, van Dissel JT, et al. 2005a. Intravitreal dexamethasone as adjuvant in the treatment of postoperative endophthalmitis: a prospective randomized trial. Graefe's Arch Clin Exp Ophthalmol, 243:1200-5.

Gan IM, Ugahary LC, van Dissel JT, et al. 2005b. Effect of intravitreal dexamethasone on vitreous vancomycin concentrations in patients with suspected postoperative bacterial endophthalmitis. Graefes Arch Clin Exp Ophthalmol, 243:1186-9.

Jett BD, Jensen HG, Atkuri RV, et al. 1995. Evaluation of therapeutic measures for treating endophthalmitis caused by isogenic toxin-producing and toxin-nonproducing Enterococcus faecalis strains. Invest Ophthalmol Vis Sci, 36:9-15.

Johnson MW, Doft BH, Kelsey SF, et al. 1997. The Endophthalmitis Vitrectomy Study. Relationship between clinical presentation and microbiologic spectrum. Ophthalmol, 104:261-72.

Kim IT, Chung KH, Koo BS. 1996. Efficacy of ciprofloxacin and dexamethasone in experimental pseudomonas endophthalmitis. Korean J Ophthalmol, 10:8-17.

Liu SM, Way T, Rodrigues M, et al. 2000. Effects of intravitreal corticosteroids in the treatment of Bacillus cereus endophthalmitis. Arch Ophthalmol, 118:803-6.

Maxwell DP Jr, Brent BD, Diamond JG, et al. 1991. Effect of intravitreal dexamethasone on ocular histopathology in a rabbit model of endophthalmitis. Ophthalmology, 98:1370-5.
Meredith TA, Aguilar HE, Drews C, et al. 1996. Intraocular dexamethasone produces a harmful effect on treatment of experimental Staphylococcus aureus endophthalmitis. Trans Am Ophthalmol Soc, 94:241-52.

Park SS, Samiy N, Ruoff K, et al. 1995. Effect of intravitreal dexamethasone in treatment of pneumococcal endophthalmitis in rabbits. Arch Ophthalmol, 113:1324-9.

Park SS, Vallar RV, Hong CH, et al. 1999. Intravitreal dexamethasone effect on intravitreal vancomycin elimination in endophthalmitis. Arch Ophthalmol, 117:1058-62.

Peyman GA, Herbst R. 1974. Bacterial endophthalmitis. Treatment with intraocular injection of gentamicin and dexamethasone. Arch Ophthalmol, 91:416-8.

Pollack JS, Beecher DJ, Pulido JS, et al. 2004. Failure of intravitreal dexamethasone to diminish inflammation or retinal toxicity in an experimental model of Bacillus cereus endophthalmitis. Curr Eye Res, 29:253-9.

Pollack JS. 2004. American Society of Retina Specialists. Preference and trends survey [Online]. Accessed 18 May 2006. URL: http://www.asrs. org/pat $/ 2004 /$ frames.php?s=75.

Shah GK, Stein JD, Sharma S, et al. 2000. Visual outcomes following the use of intravitreal steroids in the treatment of postoperative endophthalmitis. Ophthalmology, 107:486-9.

Smith MA, Sorenson JA, D’Aversa G, et al. 1997. Treatment of experimental methicillin-resistant Staphylococcus epidermidis endophthalmitis with intravitreal vancomycin and intravitreal dexamethasone. $J$ Infectious Dis, 175:462-6.

Smith MA, Sorenson JA, Smith C, et al. 1991. Effects of intravitreal dexamethasone on concentration of intravitreal vancomycin in experimental methicillin-resistant Staphylococcus epidermidis endophthalmitis. Antimicrob Agents Chemother, 35:1298-302.

West ES, Behrens A, McDonnell PJ, et al. 2005. The incidence of endophthalmitis after cataract surgery among the U.S. medicare population increased between 1994 and 2001. Ophthalmol, 112:1388-94.

Yildirim O, Oz O, Aslan G, et al. 2002. The efficacy of intravitreal levofloxacin and intravitreal dexamethasone in experimental Staphylococcus epidermidis endophthalmitis. Ophthal Res, 34:349-56.

Yoshizumi MO, Lee GC, Equi RA, et al. 1998. Timing of dexamethasone treatment in experimental Staphylococcus aureus endophthalmitis. Retina, 18:130-5. 
